\title{
NONINVASIVE ASSESSMENT OF THE VISCOELASTICITY OF PERIPHERAL ARTERIES
}

\author{
Yio-Wha Shau ${ }^{\dagger}$ Chung-Li Wang, ${ }^{\ddagger}$ Jeng-Yi ShIEH, $^{*}{ }^{\text {TsZ-ChIng Hsu }}{ }^{\S}$ \\ 'Institute of Applied Mechanics, National Taiwan University, Taipei, Taiwan, ROC; ${ }^{*}$ Department of Orthopedic \\ Surgery and *Department of Physical Medicine and Rehabilitation, National Taiwan University Hospital, Taipei, \\ Taiwan, ROC; and ${ }^{\S}$ Department of Physical Medicine and Rehabilitation, Chang Gung Memorial Hospital, Taoyuan \\ Hsien, Taiwan, ROC
}

(Received 23 March 1999; in final form 13 July 1999)

\begin{abstract}
Currently used methods of examining the mechanical properties of blood vessel walls are either indirect or invasive, or measure vessel diameter and pressure waveforms at different sites. We developed a noninvasive technique to assess the mechanical properties and viscoelasticity of peripheral arteries. The pressurestrain elastic modulus (Ep) and the viscoelastic properties (energy dissipation ratio, EDR) of the common carotid artery (CCA), brachial artery (BA), radial artery (RA) and dorsalis pedis artery (DPA) were determined by means of palpating pressure and diameter distension waveforms extracted from high-resolution ultrasonography. The methodology was validated in vitro using an elastic tube phantom, as well as in vivo. In vivo study in 10 healthy volunteers (mean age $22 \mathrm{y}$ ) showed that the pressure-diameter curves were nonlinear, with an inflection at about $85-90 \mathrm{mmHg}$, and routed clockwise with slight hysteresis. The CCA $(n=5)$ had a mean diameter of 6.74 $\mathrm{mm}$ and the pulsatile diameter distension was $12.2 \%$. The Ep calculated at the CCA was $0.44 \times 10^{6} \mathrm{dyne}^{\mathrm{cm}} \mathrm{cm}^{2}$ with an EDR of 7.18\%. The BA, RA and DPA $(n=10)$ had mean diameters of $3.91 \mathrm{~mm}, 2.21 \mathrm{~mm}$ and $2.12 \mathrm{~mm}$; arterial strains of $4.60 \%, 4.25 \%$ and $8.91 \%$; mean Ep of 1.39, 1.45, $0.90 \times 10^{6} \mathrm{dyne} / \mathrm{cm}^{2}$; and mean EDRs of $6.34 \%, 6.15 \%$ and $5.60 \%$, respectively. The method presented is relatively simple to implement clinically and has potential as a new diagnostic tool for detecting local vascular changes. (c) 1999 World Federation for Ultrasound in Medicine \& Biology.
\end{abstract}

Key Words: Ultrasound, Peripheral artery, Mechanical properties, Elastic modulus, Energy dissipation, Hysteresis.

\section{INTRODUCTION AND LITERATURE}

Understanding the changes in the dynamics of blood circulation associated with atherosclerosis is important both physiologically and clinically, and requires detailed experimental studies of local mechanical properties of vessel walls and blood flow. The effects of atherosclerosis, diabetic mellitus and hypertension on the mechanical properties of blood vessels have been reviewed extensively (Alva et al. 1993; Asmar et al. 1995; Hickler 1990; Lehmann et al. 1993a, 1997; Nerem 1992). However, most of the data in previous studies were obtained indirectly through measurement of arterial wall compliance or regional pulse wave velocity (PWV), in vitro or in vivo, with surgical exposure of the vessel (Bergel

Address correspondence to: Chung-Li Wang, M.D., Ph.D., Associate Professor, Department of Orthopedic Surgery, College of Medicine, National Taiwan University, 7, Chung Shan South Road, Taipei, 10016, Taiwan. E-mail: chungli@ha.mc.ntu.edu.tw
1961; Finkelstein et al. 1988; Lawton 1955; McDonald 1968; Patel and Janicki 1970; Peterson et al. 1960; van Loon et al. 1977).

Because exposure of blood vessels may affect their behavior, highly precise measurements of segmental arterial properties (compliance or PWV) have relied on intravascular catheterization (Gillessen et al. 1995; Ramsey et al. 1995). Advances in ultrasound (US) have made real-time noninvasive measurements of arterial diameter possible. Arndt et al. (1968) first applied a US echo device to investigate the pulsatile changes in the diameter of the common carotid artery (CCA) intact and found the elastic modulus (Ep) thus calculated to be smaller than that obtained from exposed arteries. Hokanson et al. (1972) adopted the same concept and designed a phaselocked echo-tracking device for sensing changes in the diameter of peripheral arteries in vivo.

US echo-tracking systems coupled with B-mode scanning have been subject to various modifications and 
are now commercially available (Diamove, Teltec, Lund, Sweden). With such systems, a vessel movement down to $8 \mu \mathrm{m}$ is detectable (Lindstrom et al. 1987), and there has been a growing interest in noninvasive quantitative analysis of arterial distension waveforms with US (Kelly et al. 1989b; Lanne et al. 1992; Sharir et al. 1993; Stadler et al. 1997). On the other hand, the palpating pressure waveform in peripheral arteries of superficial sites can be measured with high-fidelity tonometry pressure transducers (Kelly et al. 1989a). Because the pressure pulse and flow propagate at the same velocity, the regional arterial PWV can be measured noninvasively by using the transit time of Doppler velocity signals or pressure waveforms detected at two sites with given separation (Dauzat et al. 1996; Kingwell et al. 1997; Lehmann et al. 1993b).

Because of the complex layered nature of arterial walls, which consist of intima, media, and adventitia layers, the mechanical properties of blood vessels vary nonlinearly with intraluminal pressure (Dobrin 1983; Milnor 1989; Tanaka and Fung 1974). Also, the proportions of these three layers vary with the size of the vessel, as well as the age and the physiological state of the patient. The aortic compliance derived from the regional PWV technique further integrates the contributions of the thickness and collagen/elastin ratio of the arterial wall, and the branching and tapering of the arterial tree. Therefore, the behavior of the arterial wall cannot be fully characterized with a single parameter such as Ep or arterial compliance. The dynamic elasticity and viscoelasticity of blood vessels, which reflect changes in vascular wall composition, may be used as additional criteria for diagnosis of vascular diseases. However, direct assessment of arterial wall properties in intact vessels requires simultaneous recording of arterial diameter and pressure at the same site.

Tardy et al. (1991) conducted a noninvasive study of the mechanical properties of peripheral arteries that was based on the arterial diameter waveforms at the brachial (BA) and radial (RA) arteries vs. the pressure waveform at the finger. The pressure and diameter data were obtained simultaneously, but whether pressure waveforms at the finger accurately reflect brachial and radial waveforms is questionable, because of the tapering and wave reflection at the periphery (Milnor 1989). Several hypotheses were proposed to justify the time-delay and correct the pressure waveforms. However, the arterial viscoelasticity was assumed negligible to eliminate the hysteresis of the loading-unloading curve.

The aim of this study was to assess the mechanical properties and viscoelasticity of intact peripheral arteries including BA, RA, dorsalis pedis artery (DPA) and CCA with reasonable accuracy, using commercially available
US equipment and high-fidelity tonometry pressure transducer.

\section{MATERIALS AND METHODS}

\section{Subjects and instrumentation}

A total of 10 healthy volunteers $(5$ men and 5 women) ages $22 \pm 2$ y were recruited for this study. Their average height was $163 \mathrm{~cm}$ (men $166 \mathrm{~cm}$, women $161 \mathrm{~cm}$ ). All subjects were normotensive and nonsmokers. Each gave informed consent to participate in the experiments after receiving a full explanation of the procedures involved. Volunteers were placed in the supine position for $10 \mathrm{~min}$, and then measurements were conducted on the right RA, right BA and both DPAs. In some cases $(n=5)$, measurements were also made at right CCA. All volunteers were asked to relax the muscle of the limb being measured and refrain from breathing during CCA measurement. The ambient temperature was kept at $22 \pm 1{ }^{\circ} \mathrm{C}$.

The study was performed with a commercially available, high-resolution US scanner (HDI 3000, ATL, Bothell, WA) with a 10-MHz linear array probe (CL10-5 $26 \mathrm{~mm}, \mathrm{ATL})$. At each measurement site, the longitudinal and transverse B-mode and Doppler-mode views of the blood vessels were first recorded to quantify the inclination angle and the diameter of the blood vessel, and the insonating M-line was then aligned perpendicular to the vessel lumen in the transverse view. Thus, the pulsatile movements of the anterior and posterior walls could be visualized continuously throughout the recording.

In the meantime, a high-fidelity miniature pressure transducer (PS-2KB, strain-gauge type, $6 \mathrm{~mm}$ in diameter, Kyowa, Tokyo, Japan) was placed along the blood vessel $1 \mathrm{~mm}$ downstream from the US probe to measure the palpating pressure of the peripheral artery. A tapered cone-cylinder made of Plexiglas was placed on the tip of the pressure transducer to reduce the contact area to 1.5 $\mathrm{mm}$ in diameter. The applanation tonometry requires pressing the transducer on the surface of blood vessel and flattening the arterial wall locally. However, overcompression of arterial wall would cause changes in vessel wall mechanics and smooth muscle activity. Thus, care was taken to make sure the arteries were only slightly compressed, and the degree of compression was recorded for pulsatile diameter correction.

Because use of a hand-held transducer often suffers from irregular contact pressure and artefacts in the M-mode image due to the relative motion between transducers and measurement site, a fixture (Fig. 1) was designed to hold the two transducers together and applied to the measurement site with an elastic bandage. 


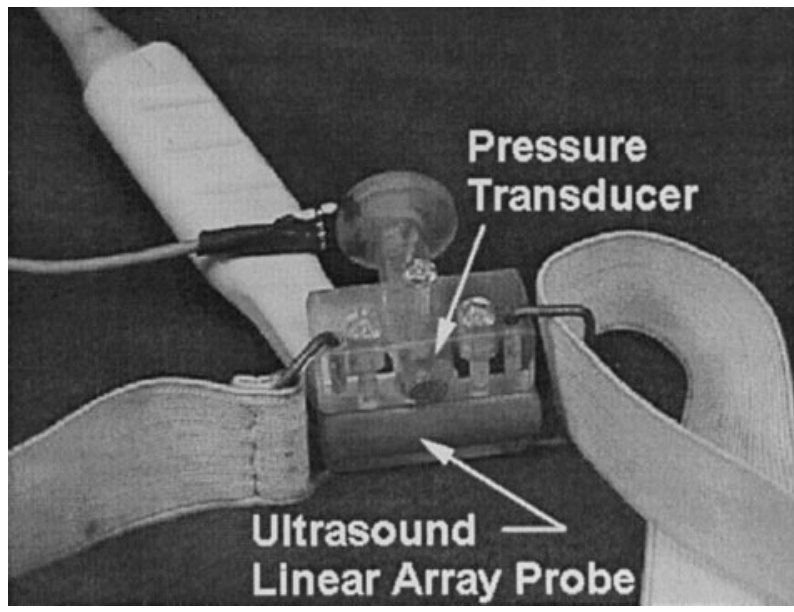

Fig. 1. Fixture design. The edge-to-edge spacing between the US linear-array probe and the pressure transducer is set at 1 $\mathrm{mm}$.

To synchronize the vessel wall movements with the palpating pressure, the output of the palpating pulse pressure was amplified and imported into the US unit so that its waveform could be displayed simultaneously with the M-mode trace on the US screen. The scanning depth, 2-D gain, and time-gain compensation (TGC) were adjusted to show a clear image of the arterial wall in both M- and B-modes. In most cases, the TGC at all depths was set to the default values or to increase linearly with depth in the region of interest.

\section{Postprocessing of digital images}

All the images captured were exported to a personal computer (IBM-compatible Intel-Pentium 200) for postprocessing. The format of the images was 640 (horizontal pixels) by 480 (vertical pixels), 8-bit or 24-bit color. An image processing software package written in $\mathrm{C}++$ was used to trace the upper and lower wall motion of vessels. On the screen, the color image was first obtained and converted to 8-bit grey-level format, and the user could then focus on the arterial wall and select the desired area for edge detection. The histogram of the grey level of the image nearby the vessel wall was analyzed. Because the acoustic impedance originating from the arterial wall is relatively constant during distension, a grey-level threshold can be selected to distinguish the outer or inner walls of vessels from the surrounding soft tissues. The location of zero crossing of the threshold along the M-line image was interpolated linearly, yielding a subpixel resolution of diameter distension waveforms. The pulse pressure recordings displayed on the screen were reviewed to exclude data with movement artefacts or marked differences in the starting amplitude of the

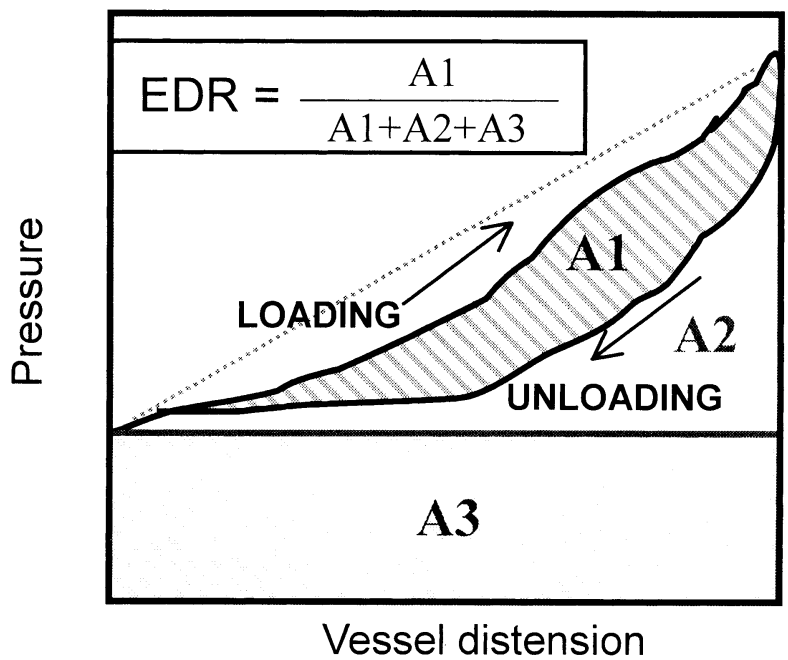

Fig. 2. Schematic of nonlinear pressure loading-unloading cycle of arterial vessel and the definition of energy dissipation ratio (EDR); the changes in pressure cause nonlinear vessel distension that depends on the pressure itself. The area A1 is mostly related to the energy dissipated due to the viscoelasticity of the vessel wall; area (A2 + A3) is the energy transmitted downstream, in which A3 is the strain energy due to initial stress (diastolic pressure).

cardiac cycle. Typically, pressure and diameter waveforms were traced during six to eight cardiac cycles, and the experimental data of two to three consecutive pressure-diameter-loading cycles were averaged. The time resolution of the palpating pressure and the diameter waveforms was set at $10 \mathrm{~ms}$. The local palpating pressure waveforms were scaled to the level of cuff systolic and diastolic pressures, as measured with an oscillometric sphygmomanometer (Dinamap Vital Sign Monitor 1856SX, Critokon, Tampa, FL). Because the direct measurement of cuff pressure at the CCA had potential risk to the brain, the CCA pulse pressure range was estimated from the systolic and diastolic pressures measured at the right $\mathrm{BA}$ and RA via sphygmomanometry. Because the pulse pressure increased gradually with the distance from the aorta along the arterial tree (Milnor 1989), the CCA pressures were extrapolated based on the distance of the $\mathrm{CCA}$ to the heart, relative to the BA and RA. The digitized waveform data were processed with a commercially available mathematical software program (Excel for Windows 95, Microsoft Co., Redmond, WA). The palpating pressure was plotted against the corresponding diameter distension at the same instant for construction of the arterial loading-unloading curve in sequential cardiac cycles (Fig. 2). The arterial strain was defined as the ratio of the pulsatile change of the inner diameter $\left(D-D_{d}\right)$ to the end-diastolic diameter $\left(D_{d}\right)$. For simplicity, the arterial Ep, adopted 


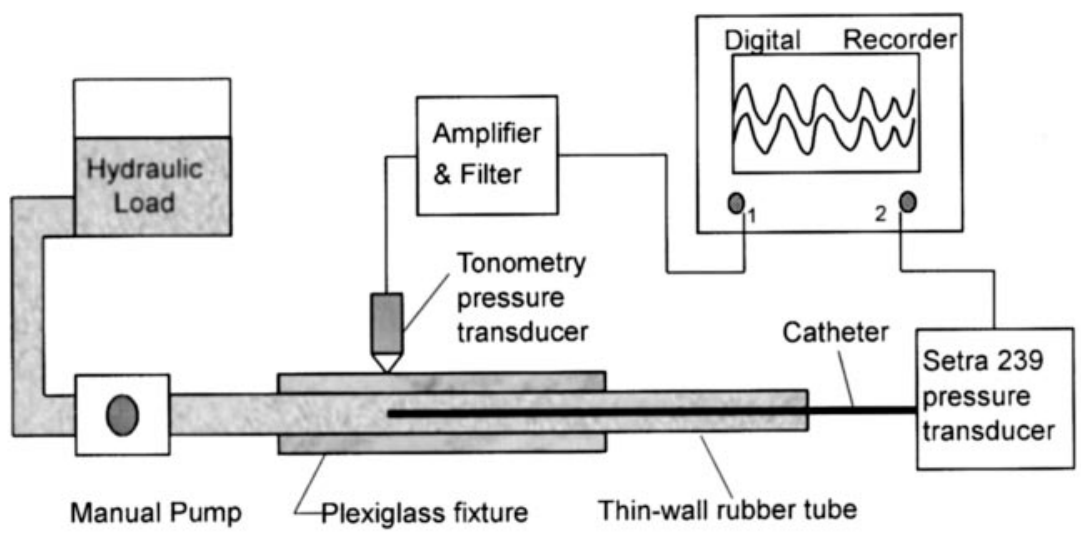

(a)
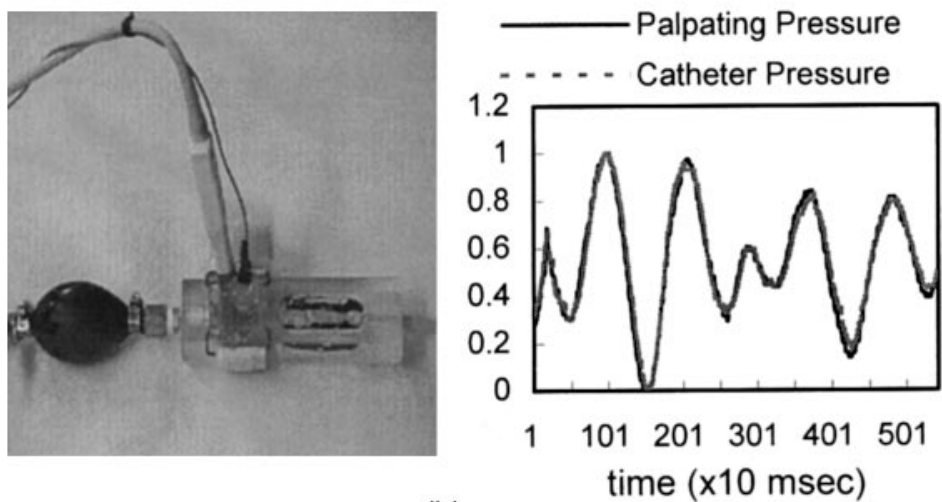

(b)

Fig. 3. In vitro verification of the palpating pressure with internal pressure measured by catheter. (a) Schematic of in vitro study using a thin-walled rubber tube phantom (tubing length $=300 \mathrm{~mm}$, wall thickness $=0.2 \mathrm{~mm}$, diameter 7.5 $\mathrm{mm}$ ). The tubing is held in a Plexiglas fixture and the catheter is inserted from the left. (b) Comparison of normalized palpating pressure and catheter pressure in phantom, recorded simultaneously with an oscilloscope. A photograph of the setup is shown on the left.

from Peterson et al. (1960), was defined as the ratio of the maximum pulsatile change in pressure to the maximum strain, $\left(D_{s}-D_{d} / D_{d}\right)$. Here, the subscript $s$ denotes the peak systolic phase and $d$ represents the end-diastolic condition. The area within the clockwise pressure-diameter loop is often related to the energy loss due to viscous dissipation in each cardiac cycle (Fung 1993). Thus, we may define an energy dissipation ratio (EDR) that denotes the ratio of the energy dissipated to the total energy imparted by blood pressure in systolic phase.

\section{In vitro validation of palpating pressure}

The response and accuracy of the tonometry pressure transducer were first verified in an in vitro study using an elastic rubber tube phantom, as shown schematically in Fig. 3a. The phantom consisted of a 300-mm long distensible tube with a wall thickness of $0.2 \mathrm{~mm}$, secured in a Plexiglas fixture. The tube was inflated with water at an initial in- traluminal pressure of about $5-10 \mathrm{mmHg}$, which gave the tubing an initial diameter of about $7.5 \mathrm{~mm}$. The elastic tube was placed horizontally with the upper wall about $10 \mathrm{~mm}$ below the surface of a US transducer, and was connected to a manual hydraulic pump. A 30-cm long stainless-steel tube with diameter of $1.2 \mathrm{~mm}$, inserted through the rear of the elastic tube, was used as a catheter to connect the elastic tubing to a high-precision pressure transducer (Model-239, range 0-76 cm WC, Setra, Acton, MA) for measurement of the intraluminal pressure at the same site. By compressing the pump repeatedly, the palpating pressure and intraluminal pressure waveforms could be recorded simultaneously with an oscilloscope (TDS-220, Tektronix, Beaverton, OR). Because the intraluminal pressure wave propagates through the rigid catheter at a finite speed, the response time-delay of catheter pressure waveform on the order of $1 \mathrm{~ms}$ was corrected (Doebelin 1990). Also, we incorporated the structural damping in the liquid-filled pressure system to minimize the oscillations in the catheter. 

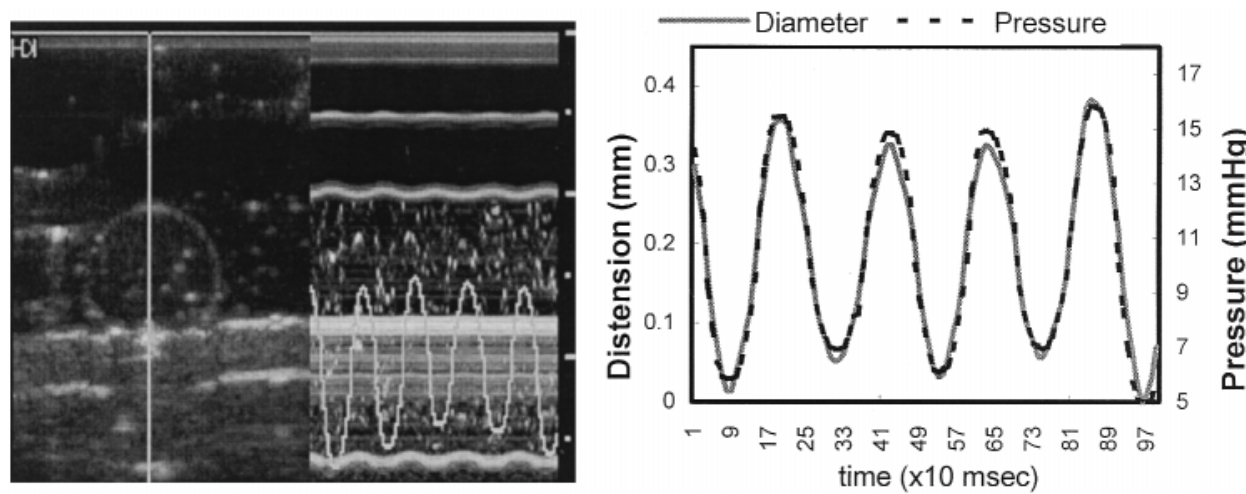

(a)

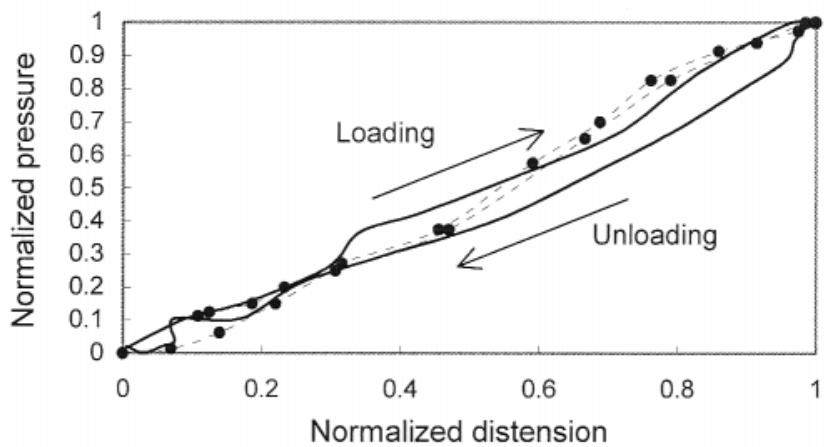

(b)

Fig. 4. In vitro validation of the study of mechanical properties using a thin-walled elastic tube. (a) Comparison of internal diameter waveforms and the palpating pressure detected from the B-M-mode image (the transverse view of the elastic tube, the wall distension, and the palpating pressure waveforms are displayed simultaneously on the left). (b) Pressure-diameter loading curves obtained with the pressure transducer contacting the tube (—with marker) or with a muscle layer (chicken breast of $1 \mathrm{~cm}$ in thickness;-) placed between the elastic tubing and both transducers. The pressure increases linearly with diameter distension. The hysteresis increases due to the viscoelasticity of the covered muscle.

In vitro validation of mechanical properties of blood vessels

The validity of the proposed method for assessing the mechanical properties of blood vessels with US and applanation tonometry was demonstrated through in vitro study with thin-walled elastic tubing. At the measurement sites, the elastic tube was covered with US couplant and the pressure transducer was attached to the tubing surface to measure the intraluminal pressure. Both the palpating pressure and wall motion of the elastic tube were also recorded simultaneously with US (Fig. 4a).

Because noninvasive measurement of mechanical properties of peripheral arteries requires sensing a vessel at a depth of about 5-10 $\mathrm{mm}$ below the skin, the results could be affected by intervening soft or fatty tissues. We, therefore, investigated the effect of soft tissue on the measurement of palpating pressure and diameter distensibility by inserting a $10-\mathrm{mm}$ thick section of chicken breast (to simulate the muscle layer, which has a higher viscoelasticity) between the transducers and the elastic tube.

\section{Statistical analysis}

All values are given as mean \pm standard deviation (SD). Student's $t$-test was used for statistical analysis. The reproducibility of the data was verified by taking the measurements at least twice at each location and measuring 3 subjects on 3 separate occasions within 2 months, with at least 7 days between visits.

\section{RESULTS}

\section{In vitro validation of the methodology}

As shown in Fig. 3b, the normalized waveforms of the palpating and catheter pressures were almost identical to each other in the frequency range of physiological heart rate.

In Fig. 4a, the echo of the wall motion generated an observable mirror artefact below the posterior wall sur- 
face. The pulsatile diameter distension waveforms detected from the M-mode image are also shown with the palpating pressure waveform. The mean inner diameter of the tubing determined with US was about 3-5\% higher than the actual size, because a stationary speed of sound (i.e., $c=1540 \mathrm{~m} / \mathrm{s}$ ) was typically used in the calculation of depth from the US echo.

The pressure-diameter curves obtained by combining the normalized pressure and diameter recordings are shown in Fig. 4b. The loading-unloading curves obtained by applying the pressure transducer directly on the tube were linear with little hysteresis (EDR of $0.2 \%$ ). This shows that the thin-walled rubber tubing is elastic with negligible viscoelasticity, as expected, and the proposed methodology is generally acceptable.

The viscoelasticity of the muscle layer increased the hysteresis of the pressure-diameter curve slightly (from $0.2 \%$ to $5.2 \%$, Fig. $4 \mathrm{~b}$ ); however, the palpating pressure waveform still resembled the catheter pressure or diameter pulsation in an elastic manner. Because muscle has higher viscous damping than collagen or elastin (Fung 1993), for the superficial arteries in our study, the hysteresis resulting from skin tissue should be smaller than that from the muscle layer applied here.

\section{In vivo demonstration of the methodology}

Figure 5a shows a representative B-M-mode image obtained at the BA. The setup used for measurement at the BA is shown in the upper left of the figure. With the aid of the US B-mode image, the blood vessel can easily be located for positioning of the pressure transducer. The BA examined was $3.14 \mathrm{~mm}$ in mean diameter, and about $5 \mathrm{~mm}$ below the skin surface. The pressure waveforms recorded next to the US transducer are displayed below in the same image. Because the local PWV is on the order of 5-10 m/s (Milnor 1989), the time-delay between the pressure waveform and diameter distension waveform is about $1 \mathrm{~ms}$. This systemic time-delay was corrected in the construction of the pressure-diameter curve.

The arterial diameter distension waveforms obtained with various edge-detection thresholds resembled each other, except in the late diastolic phase (Fig. 5b). The diameter distension waveforms could be detected from the grey-level histogram nearby the vascular wall, so that the results were insensitive to the threshold within the range of optimum selection. For a mean BA diameter of $3.14 \mathrm{~mm}$, the maximum distension of diameter was about $0.24 \mathrm{~mm}$, which corresponded to a maximal strain of $7.64 \%$. The diameter distension waveforms for six cardiac cycles were plotted, together with the pulse pressure waveforms (dashed line) scaled to the systolic and diastolic pressures from cuff measurements taken at the same location. In the systolic phase, the diameter followed the pressure very well. However, in the diastolic phase of the cardiac cycle, the diameter lagged slightly behind, resulting in a small hysteresis in the clockwise pressure diameter curves (Fig. 5c). The data curves followed roughly the same path, and demonstrated good reproducibility. Also, the change of Ep with respect to blood pressure could be determined by normalizing the diameter distension with the diastolic arterial diameter. The Ep was relatively constant, about $1.22 \times 10^{6}$ dyne/ $\mathrm{cm}^{2}$ in this subject, and the EDR was about $4.2 \%$.

Figure 6a shows the setup used for measurement at the CCA, along with the US B-M-mode image. The anterior-posterior wall displacements of the CCA are shown in Fig. 6b. The anterior wall and posterior wall reacted to the pulsatile pressure differently because of differences in the stiffness of the soft tissue surrounding the blood vessel. The blood vessel deflected upward in the third cycle; however, the inner diameter as calculated from the difference of the two waveforms was not affected. The corresponding simultaneously recorded blood pressure waveforms were digitized and scaled to approximately the values of the systolic and diastolic cuff pressures. The resulting pressure-diameter loadingunloading curve of the CCA is shown in Fig. 6c. The Ep determined from the slope was nearly constant, $0.43 \times$ $10^{6}$ dyne $/ \mathrm{cm}^{2}$ in this case, and the EDR was about $6.52 \%$. A deflection of the pressure-diameter curve at 85-90 mmHg was observed.

The pressure and inner diameter waveforms measured simultaneously at the RA are shown in Fig. 7a. The corresponding pressure-diameter curves for seven cardiac cycles that marked the nonlinear behavior of the RA can be seen in Fig. 7b. Similarly, typical pressure and diameter distension waveforms obtained simultaneously at the DPA are plotted in Fig. 8a and the clockwise loading-unloading curve is shown in Fig. 8b. In both Figs. 7 and 8, the diameter distension waveforms resemble the pulsatile pressure curves, and the deflection in the pressure-diameter curves at about $85-90 \mathrm{mmHg}$ can also be seen.

Table 1 summarizes the mechanical properties measured at intact peripheral arteries for healthy subjects in the supine position. The coefficients of variation were less than $5 \%$ for the diameter and systolic and diastolic pressure measurements and less than $6 \%$ for the diameter distension and pressure waveform values determined from various thresholds and phantom calibration. The EDR value was affected by the phase-delay of the two waveforms and soft tissue viscous damping and had a larger uncertainty, typically about $\pm 3 \%$ of the total strain energy.

\section{DISCUSSION}

Arteries belong to a class of soft tissues that exhibit nonlinear stress-strain relationships and hysteresis during cyclic loading and unloading (Fung 1993). The hystere- 


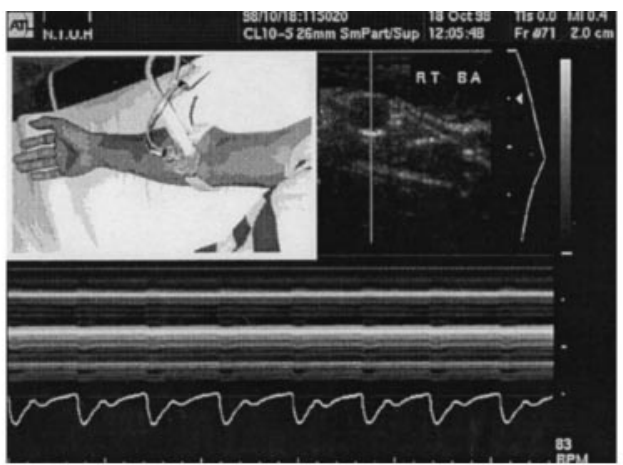

(a)

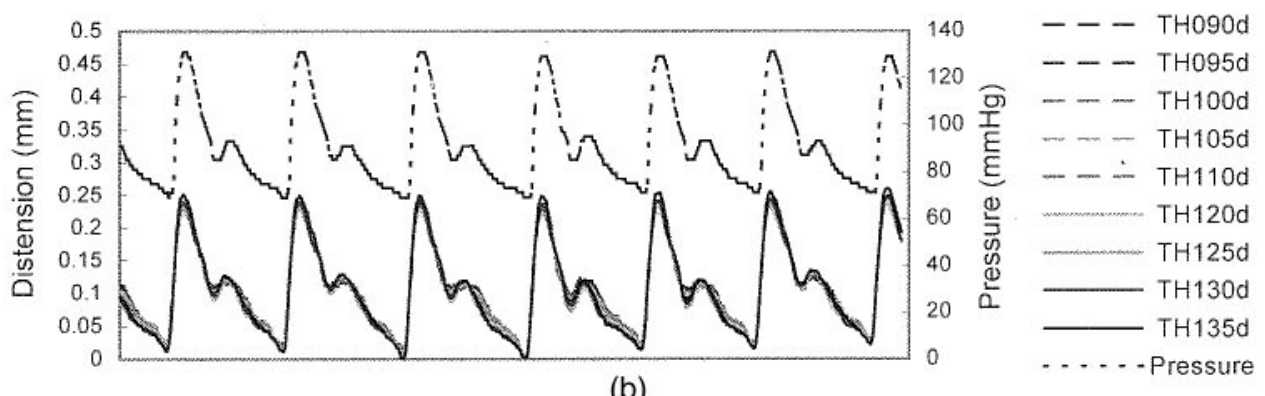

(b)

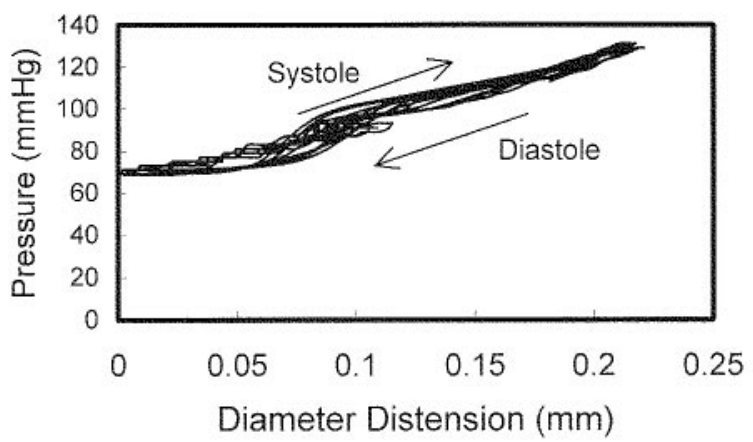

(c)

Fig. 5. Representative pressure-diameter curves obtained at the brachial artery (BA) in a healthy young subject (male, age $23 \mathrm{y}$, BA diameter $3.14 \mathrm{~mm}$ ). (a) US B/M-mode image with palpating pulse pressure waveform displayed at the bottom (in this setting, the time scale $1 \mathrm{~s}=100 x$-pixels, and length scale $1 \mathrm{~cm}=99 y$-pixels) The experimental setup for measurement of mechanical properties at the BA is shown at the upper left of the figure. (b) Effect of thresholds on the diameter distension waveforms of the BA detected from (a); the optimum threshold determined from the grey-level histogram is $\mathrm{TH}=105 \mathrm{~d}$. For a mean BA diameter of $3.14 \mathrm{~mm}$, the maximum strain is about $7.64 \%$. The palpating pressure waveform of the BA detected simultaneously using (a) is also shown. (c) Nonlinear pressure-diameter curves obtained by combining the pressure and diameter recorded in (b). The results showed slight hysteresis in the clockwise pressure loading-unloading cycle for the BA. Experimental data for six consecutive cardiac cycles are plotted and show a good reproducibility.

sis in the clockwise pressure-diameter relationship indicates that both elastin and collagen contribute to the mechanical properties of arteries (Dobrin 1983; Lanne et al. 1992). Although arterial properties have been studied for decades, only in recent years have various noninvasive techniques been developed to depict the characteristics of the dynamic motion of arterial walls realistically. Laurent et al. (1994) measured noninvasively the
CCA pressure-diameter curve by recording simultaneously the pulsatile changes of internal diameter and pressure waveforms using echo-tracking US and applanation tonometry, respectively. The elastic response of the blood vessel was investigated through the distensibility- and compliance-pressure curves. We find the viscoelasticity (energy dissipation) exists in their CCA pressure-diameter results. The method described herein al- 


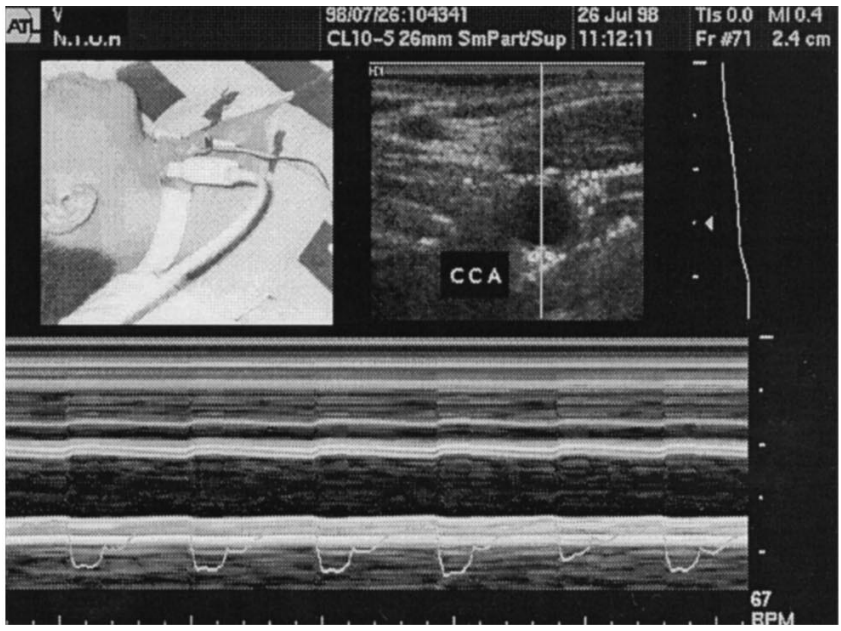

(a)

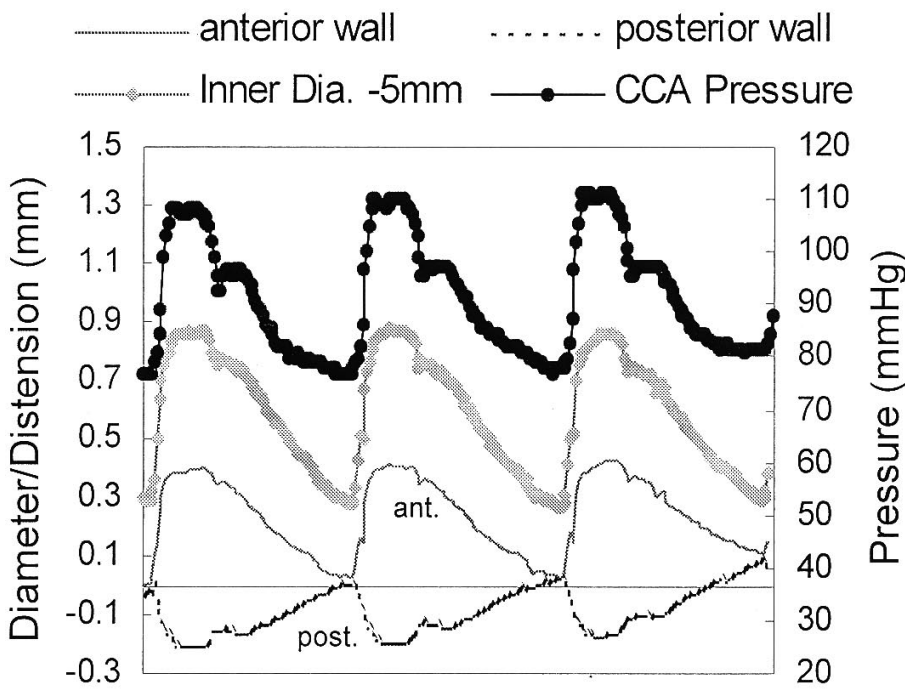

(b)

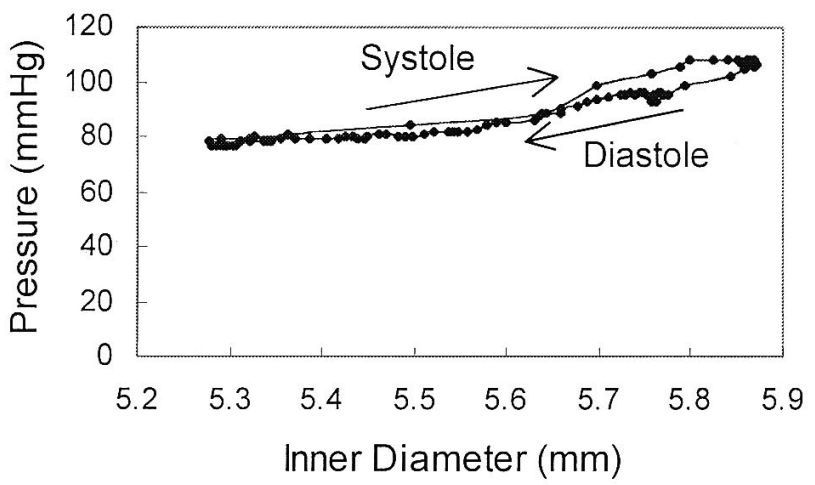

(c)

Fig. 6. Representative data obtained from common carotid artery (CCA) of a young healthy subject (female, age 23 y, diameter $=5.69 \mathrm{~mm}$ ). (a) B-M-mode US image of CCA. The experimental setup for measurement at the CCA is shown at the upper left of the figure. (b) Anterior, posterior wall distensions and CCA inner diameter waveforms for three cardiac cycles; the wall distension waveforms show similar profiles. The palpating pressure waveforms at CCA were scaled values extrapolated from the systolic and diastolic pressures measured at BA and RA based on their distances to the heart. (c) Pressure-diameter curve at the CCA obtained from (b). 


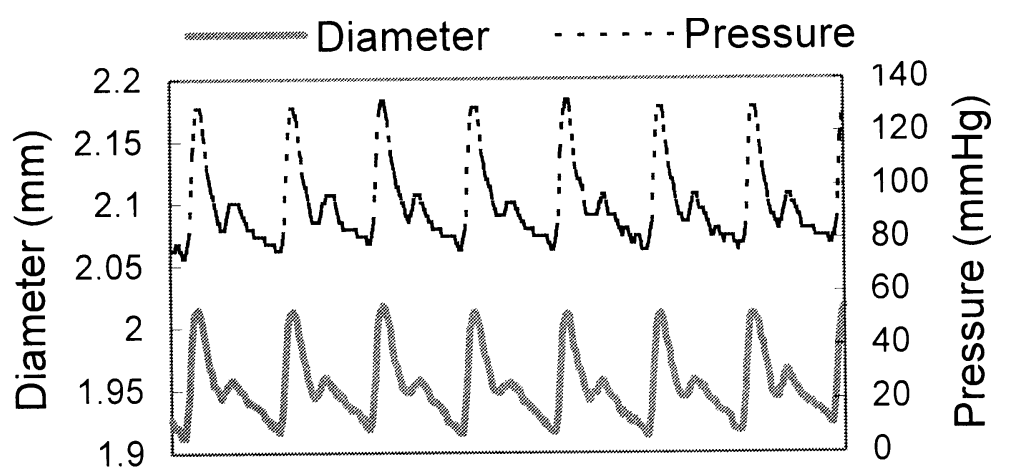

(a)

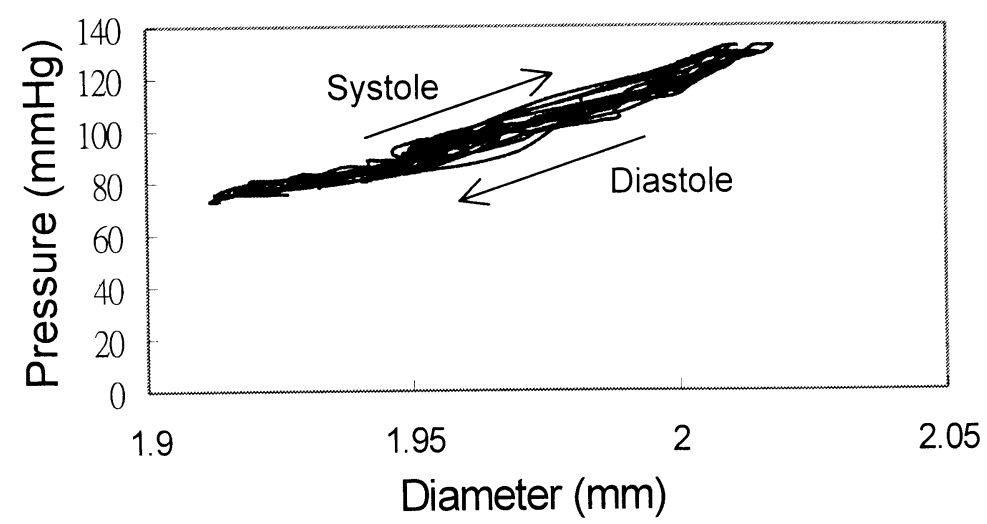

(b)

Fig. 7. Representative data obtained from the radial artery (RA) of a healthy young subject (female, age 23 y, mean diameter $1.98 \mathrm{~mm}$ ). (a) Simultaneous recording of diameter and pressure waveforms from RA. (b) Clockwise pressure diameter loading curve at the RA.

lows noninvasive assessment of viscoelasticity in the pressure-diameter relationship in intact peripheral arteries (CCA, BA, RA and DPA).

The B-M-mode clinical US, coupled with highfidelity tonometry, allows accurate determination of the pressure-diameter curve of superficial arteries. This noninvasive method can depict pressure waveforms satisfactorily. Although the tonometry pressure transducer was calibrated to provide absolute pressure change, to avoid the attenuation of pressure due to soft tissue covering the blood vessel, the pulse pressures (systolic and diastolic pressure values) relied on sphygmomanometry. Our experiments with the thin-walled elastic tube phantom proved this method to be reliable and accurate.

The clinical US device (HDI-3000) used in this study has a built-in image processing function for improving the image displayed on-screen. We found that digital filtering or curve fitting was not necessary to obtain a smooth diameter distension.

The diameter distension waveforms measured from the B-M-mode images are relatively independent of the threshold used in edge-detection, unless the TGC is adjusted improperly. A smooth distension waveform on the order of $10 \mu \mathrm{m}$ that resembles the pressure waveform was still detectable at the RA in this study. The motion of the soft tissues and blood vessel can be monitored continuously with our edge-detection technique, although the resolution of the diameter distension is slightly less than with the echo-tracking system. Moreover, the diameter distension waveforms detected in the M-mode image should be corrected for the longitudinal angle of inclination to improve accuracy.

One of the main advantages of the present method over traditional measurements of the PWV is that the pressure and diameter of the blood vessel are measured simultaneously at the same location, so that local vascular changes can be assessed. Also, because the degree of compression of blood vessel in using applanation tonometry can be visualized transversely on-screen, the palpating pressure waveforms obtained are self-similar unless the change in vessel diameter exceeds $15 \%$ of its natural size. In the present system, overcompression of the superficial arteries can be easily avoided.

Cardiovascular disorders are often associated with 


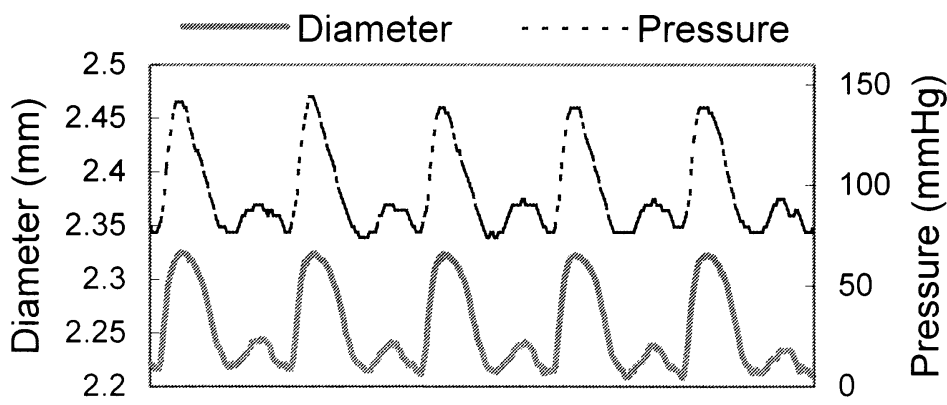

(a)

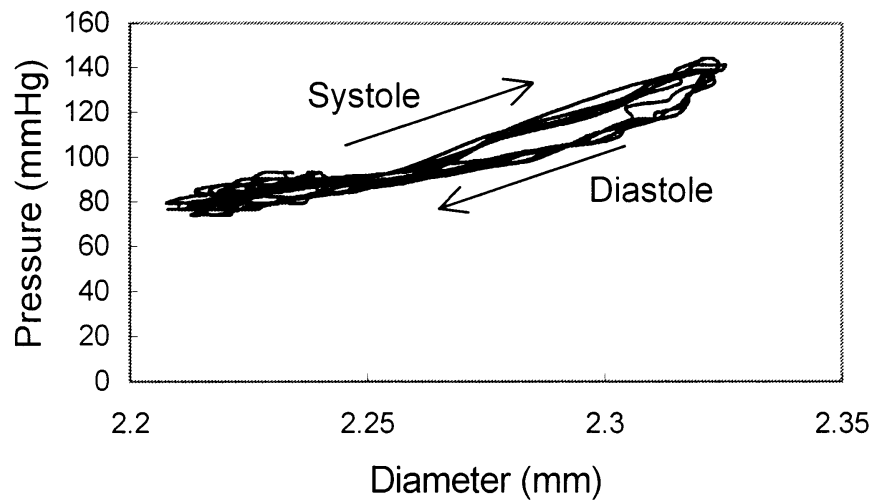

(b)

Fig. 8. Representative data obtained from the dorsalis-pedis artery (DPA) of a healthy young subject (female, age $23 \mathrm{y}$, diameter $=2.28 \mathrm{~mm}$ ). (a) Simultaneous recording of diameter and pressure waveforms at the DPA. (b) Clockwise pressure diameter loading curve for one cardiac cycle at the DPA.

arterial stiffening or changes in arterial composition (Hansen et al. 1995), and this may result in changes in Ep, loading-unloading curves, and EDR. The pressurestrain curve of the arterial wall has been described as an exponential function of wall stiffness:

$$
\beta=\log \frac{P_{s} / P_{d}}{\left(D_{s}-D_{d}\right) / D_{d}}
$$

where $\beta$ is stiffness, $P$ represents pressure, and $D$ represents vessel diameter (Hansen et al 1995; Lehmann et al. 1993a). In our study, the Ep value of the CCA was $0.44 \times 10^{6}$ dyne $/ \mathrm{cm}^{2}$ and the $\beta$ value was 3.6 ; these values fall within the range of those reported in the literature (Hansen et al. 1995). By fitting the normalized pressure-diameter curves with mathematical functions, the loading and unloading phases would give two different values.

Because the present method is performed on intact superficial arteries, the stress-strain relationship of the blood vessels is repeatable and no preconditioning is needed. However, this method is applicable for

Table 1. Mechanical properties of peripheral arteries in healthy subjects

\begin{tabular}{|c|c|c|c|c|c|c|}
\hline Artery & $\begin{array}{c}P_{s} \\
(\mathrm{mmHg})\end{array}$ & $\begin{array}{c}P_{d} \\
(\mathrm{mmHg})\end{array}$ & $\begin{array}{l}\text { Diameter } \\
(\mathrm{mm})\end{array}$ & $\begin{array}{c}\text { Strain } \\
\left(D_{s}-D_{d}\right) / D_{d},(\%)\end{array}$ & $\begin{array}{c}\text { Ep } \\
\left(10^{6} \text { dyne } / \mathrm{cm}^{2}\right)\end{array}$ & $\operatorname{EDR}(\%)$ \\
\hline CCA & $112.2(6.5)$ & $72.6(3.1)$ & $6.74(0.87)$ & $12.17(1.23)$ & $0.439(0.083)$ & $7.18(1.84)$ \\
\hline BA & $118.0(10.7)$ & $69.8(5.3)$ & $3.91(0.59)$ & $4.60(1.12)$ & $1.390(0.240)$ & $6.34(1.45)$ \\
\hline RA & $121.0(11.7)$ & $75.4(7.9)$ & $2.21(0.39)$ & $4.25(1.30)$ & $1.447(0.357)$ & $6.15(2.37)$ \\
\hline DPA & $132.3(10.3)$ & $70.5(6.3)$ & $2.12(0.37)$ & $8.91(0.88)$ & $0.903(0.109)$ & $5.60(1.29)$ \\
\hline
\end{tabular}

$P_{s}=$ systolic pressure; $P_{d}=$ diastolic pressure; $D_{s}=$ peak systolic diameter; $D_{d}=$ end-diastolic diameter; Ep $=$ pressure-strain elastic modulus; $\mathrm{EDR}=$ energy dissipation ratio; $\mathrm{CCA}=$ common carotid artery; $\mathrm{BA}=$ brachial artery; $\mathrm{RA}=$ radial artery; DPA $=$ dorsalis-pedis artery. $\mathrm{BA}$ Ra DPA data are mean (standard variation) of 10 volunteers ( 5 men, 5 women, mean age 22 y). CCA data were collected from 5 subjects ( 3 men, 2 women, mean age $22 \mathrm{y}$ ). 
assessment of superficial arteries only because the reliability of palpating pressure data would be inadequate in deeper vessels. In principle, a flattening surface of applanation tonometer allows direct measurements of internal pressure of blood vessels. In this study, a pressure sensing area of $1.5 \mathrm{~mm}$ in diameter is smaller than the size of peripheral arteries examined. However, its sharpness may become problematic in diabetic patients, whose skin is fragile and vulnerable to injury during the test. Moreover, for blood vessels at greater depths, tonometry pressure can be used only as an approximation, because the high-frequency contents of the intraluminal pressure would be smeared by the soft tissue lying between, and the uncertainty in hysteresis would be increased also. The present system specially features the energy dissipation ratio of peripheral arteries and it should be suitable for investigating the effects of physiological and pharmacological stimuli on the peripheral arteries.

\section{SUMMARY}

The noninvasive technique described herein incorporates a commercially available US device and applanation tonometry to assess the mechanical properties of local peripheral arteries intact. Measurements were made at the CCA, BA, RA and DPA in a group of healthy young subjects $(n=10 ; 5$ male, 5 female; mean age $22 \mathrm{y}$ ). The palpating pressure and diameter distension waveforms were recorded and displayed simultaneously on the B-M-mode image of a highresolution US device, and the Ep and EDR were then determined. The methodology was validated in vitro using a thin-walled elastic tube. In normal physiological pressure ranges, the CCA data agreed with those reported in the literature. The DPA was more distensible than the BA or RA. The EDR in the peripheral arteries of our volunteers was relatively small, in the range of $3-10 \%$. The results demonstrated that the pressure-diameter curve, as well as the viscoelasticity, of peripheral artery walls can be determined noninvasively with reasonable accuracy. We conclude that the method presented in this study is relatively simple to implement and has potential as a new diagnostic tool for detecting local vascular changes. In the future, it may prove useful for clinical diagnosis of early degeneration of blood vessel due to cardiovascular diseases.

\section{REFERENCES}

Alva F, Samaniego V, Gonzalez V, Moguel R, Meaney E. Structural and dynamic changes in the elastic arteries due to arterial hypertension and hypercholesterolemia. Clin Cardiol 1993;16:614-618. Arndt JO, Klauske J, Mersch F. The diameter of the intact carotid artery in man and its change with pulse pressure. Pflugers Arch 1968;301: $230-240$.

Asmar R, Benetos A, Topouchian J, et al. Assessment of arterial distensibility by automatic pulse wave velocity measurement. Validation and clinical application studies. Hypertension 1995;26:485-490.

Bergel DH. The dynamic elastic properties of the arterial wall. J Physiol 1961;156:458-469.

Dauzat M, Deklunder G, Adam B, et al. Pulse wave velocity measurement by cross-correlation of Doppler velocity signals. Application to elderly volunteers during training. Int J Sports Med 1996;17: 547-553.

Dobrin P. Vascular mechanics. In: Shepard JT, Abboud FM, eds. Handbook of physiology. Part I, Peripheral circulation and organ blood flow. Baltimore, MD: Williams \& Wilkins, 1983:65-102.

Doebelin EO. Measurement system: application and design. 4th ed. Singapore: McGraw-Hill, 1990.

Finkelstein SM, Collins VR, Cohn JN. Arterial vascular compliance response to vasodilators by Fourier and pulse contour analysis. Hypertension 1988;12:380-387.

Fung YC. Biomechanics: Mechanical properties of living tissues. 2nd ed. New York: Springer-Verlag, 1993.

Gillessen T, Gillessen F, Sieberth H, Hanrath P, Heintz B. Age-related changes in the elastic properties of the aortic tree in normotensive patients: investigation by intravascular ultrasound. Eur J Med Res 1995; $1: 144-148$.

Hansen F, Mangell P, Sonesson B, Lanne T. Diameter and compliance in the human common carotid artery-variations with age and sex. Ultrasound Med Biol 1995;21:1-9.

Hickler RB. Aortic and large artery stiffness: current methodology and clinical correlations. Clin Cardiol 1990;13:317-322.

Hokanson DE, Mozersky DJ, Sumner DS, Strandness DE Jr. A phaselocked echo tracking system for recording arterial diameter changes in vivo. J Appl Physiol 1972;32:728-733.

Kelly R, Daley J, Avolio A, O'Rourke M. Arterial dilation and reduced wave reflection. Benefit of dilevalol in hypertension. Hypertension $1989 \mathrm{a} ; 14: 14-21$.

Kelly R, Hayward C, Avolio A, O'Rourke M. Noninvasive determination of age-related changes in the human arterial pulse. Circulation $1989 \mathrm{~b} ; 80: 1652-1659$.

Kingwell BA, Berry KL, Cameron JD, Jennings GL, Dart AM. Arterial compliance increases after moderate-intensity cycling. Am J Physiol 1997;273:H2186-H2191.

Lanne T, Stale H, Bengtsson H, et al. Noninvasive measurement of diameter changes in the distal abdominal aorta in man. Ultrasound Med Biol 1992;18:451-457.

Laurent S, Caviezel B, Beck L, Girerd X, Billaud E, Boutouyrie P, Hoeks A, Safar M. Carotid artery distensibility and distending pressure in hypertensive humans. Hypertension 1994;23(2):878-883.

Lawton RW. Measurements on the elasticity and damping of isolated aortic strips of the dog. Circ Res 1955;3:403-408.

Lehmann ED, Hopkins KD, Gosling RG. Aortic compliance measurements using Doppler ultrasound: in vivo biochemical correlates. Ultrasound Med Biol 1993a;19:683-710.

Lehmann ED, Parker JR, Hopkins KD, Taylor MG, Gosling RG. Validation and reproducibility of pressure-corrected aortic distensibility measurements using pulse-wave-velocity Doppler ultrasound. IEEE Trans Biomed Eng 1993b;15:221-228.

Lehmann ED, Riley WA, Clarkson P, Gosling RG. Non-invasive assessment of cardiovascular disease in diabetes mellitus. Lancet 1997;350(Suppl. 1):SI14-S119.

Lindstrom K, Gennser G, Sindberg EP, Bentin M, Dahl P. An improved echo-tracker for studies on pulse waves in fetal aorta. In: Rolfe P, ed. Fetal physiological measurements. London: Butterworths, 1987:217-226.

McDonald DA. Regional pulse-wave velocity in the arterial tree. J Appl Physiol 1968;24:73--78.

Milnor WR. Hemodynamics. 2nd ed. Baltimore, MD: Williams \& Wilkins, 1989.

Nerem RM. Vascular fluid mechanics, the arterial wall, and atherosclerosis. J Biomech Eng 1992;114:274-282.

Patel DJ, Janicki JS. Static elastic properties of the left coronary 
circumflex artery and the common carotid artery in dogs. Circ Res 1970;27:149-158.

Peterson LH, Jenson RE, Parnell J. Mechanical properties of arteries in vivo. Circ Res 1960;8:622-639.

Ramsey MW, Stewart WR, Jones CJ. Real-time measurement of pulse wave velocity from arterial pressure waveforms. Med Biol Eng Comput 1995;33:636-642.

Sharir T, Marmor A, Ting CT, et al. Validation of a method for noninvasive measurement of central arterial pressure. Hypertension 1993;21:74-82.

Stadler RW, Taylor JA, Lees RS. Comparison of B-mode, M-mode and echo-tracking methods for measurement of the arterial distension waveform. Ultrasound Med Biol 1997;23:879-887.

Tanaka TT, Fung YC. Elastic and inelastic properties of the canine aorta and their variation along the aortic tree. J Biomech 1974;7: 357-370.

Tardy Y, Meister JJ, Perret F, Brunner HR, Arditi M. Non-invasive estimate of the mechanical properties of peripheral arteries from ultrasonic and photoplethysmographic measurements. Clin Phys Physiol Meas 1991;12:39-54.

van Loon P, Klip W, Bradley EL. Length-force and volume-pressure relationships of arteries. Biorheology 1977;14:181-201. 